\title{
Germanica
}

\section{La folie par contamination Littérature et schizophrénie dans un roman de Simon Vestdijk}

Geisteskrankheit durch Übertragung, Literatur und Schizophrenie in einem

Roman von Simon Vestdijk

Spiros Macris

\section{(2) OpenEdition}

Journals

Édition électronique

URL : http://journals.openedition.org/germanica/1847

DOI : 10.4000/germanica. 1847

ISSN : 2107-0784

Éditeur

Université de Lille

\section{Édition imprimée}

Date de publication : 30 juin 2003

Pagination : 61-75

ISBN : 9782913857117

ISSN : 0984-2632

Référence électronique

Spiros Macris, "La folie par contamination Littérature et schizophrénie dans un roman de Simon Vestdijk », Germanica [En ligne], 32 | 2003, mis en ligne le 07 décembre 2012, consulté le 06 octobre 2020. URL : http://journals.openedition.org/germanica/1847 ; DOI : https://doi.org/10.4000/ germanica. 1847

Ce document a été généré automatiquement le 6 octobre 2020.

(c) Tous droits réservés 


\section{La folie par contamination Littérature et schizophrénie dans un roman de Simon Vestdijk}

Geisteskrankheit durch Übertragung, Literatur und Schizophrenie in einem

Roman von Simon Vestdijk

Spiros Macris

1 Dans la marge d'un cahier où il note, en janvier 1945, une vingtaine de sujets de romans ou de nouvelles, Simon Vestdijk (1898-1971) ajoute une brève mention : " 3 hommes nus, D. Margadant ». Dick Margadant était un cousin germain de cinq ans son aîné qui souffrait de schizophrénie ${ }^{1}$. Ces quelques mots annoncent le roman De redding van Fré Bolderhey (Le salut de Fré Bolderhey) ${ }^{2}$, écrit en 1948, dont le thème est la progression de la schizophrénie chez un jeune garçon de 16 ans fasciné par un cousin germain plus âgé, schizophrène avéré.

2 L'étude du fou dans la littérature évoque en premier lieu une série de portraits, et le héros malheureux de ce roman y trouve une place d'autant plus assurée que son tableau clinique a, depuis, reçu l'aval de la psychiatrie. Le regard de Vestdijk est en effet informé à plus d'un titre. Après des études de médecine, il exerce plusieurs années, notamment comme médecin de bord. Cette activité a aiguisé l'esprit analytique qui marque ses poèmes, romans et essais. Cette disposition doit être mise en relation avec l'expérience qu'il fait de la fragilité humaine. Depuis l'âge de 17 ans, il est régulièrement frappé de dépressions. Durant ces crises presque annuelles il doit se retirer du monde, incapable de tout travail soutenu. L'échec des traitements les plus divers, de l'opium aux électrochocs en passant par la psychanalyse à laquelle il s'est montré rétif, est lié à la conviction de l'écrivain que l'angoisse est le moteur de sa création.

3 Ces circonstances n'auraient en soi guère d'intérêt si elles ne jouaient un rôle dans l'économie de l'œuvre. Dans Het wezen van de angst (L'essence de l'angoisse), une thèse volumineuse entreprise pour des raisons principalement thérapeutiques et rédigée en 
quelques mois, la même année que Fré Bolderhey, Vestdijk note : « Dans la beauté, l'objet de l'angoisse est objectivé à un tel degré que nous ne pouvons que l'admirer, et non plus le redouter. Mais face à tout mysterium tremendum l'admiration retrouve le chemin de l'angoisse et, confronté aux pires menaces, le sens du beau [schoonheidszin] reste impuissant $»^{3}$. Dans cette lutte incertaine, tous les alliés sont requis pour combattre l'ennemi intime: un matériau autobiographique largement exploité et refondu, une discipline rigoureuse, les armes de l'intellect et un solide prosaïsme mis au service d'une force créatrice prodigieuse. Il est entré en littérature vers 34 ans et a dû faire face à des périodes d'inactivité forcées. C'est donc sur une durée relativement courte que l'auteur a produit une œuvre immense ${ }^{4}$ dont les 52 romans représentent la part essentielle. Vestdijk est un auteur méthodique, il planifie, fait des fiches, se documente avec soin et attache une grande importance à la technique littéraire. Il occupe avec un haut degré de conscience trois positions : celle du patient, du médecin et de l'écrivain. Chaque posture est à la fois authentique et stratégique vis-à-vis des deux autres, créant un système que De redding van Fré Bolderhey permet d'éclairer.

La schizophrénie est, en 1948, une maladie assez récente. Soucieux d'ordonner les désordres mentaux selon une classification générale et raisonnée, Emile Kraepelin (1856-1926) avait rassemblé à partir de 1883 sous le nom «démence précoce » les atteintes touchant surtout de jeunes gens et dont l'évolution paraissait irréversible. Comme l'ensemble de la nosographie, cette catégorie regroupe des manifestations très hétérogènes. Elle est fondée sur la notion de psychose endogène liée à une atteinte neurologique laquelle fait du malade un être fermé sur lui-même. Il est la seule cause de sa maladie et n'a d'autre perspective que l'internement pour qu'il en reste la seule victime. Eugen Bleuler (1857-1939) s'opposera à cette approche en insistant sur la dimension affective et relationnelle de la maladie pour laquelle il crée en 1911 le terme de schizophrénie. C'est désormais la structuration psychique qui est privilégiée. L'idée centrale de clivage ou de dissociation (Spaltung) existait déjà mais désignait les cas de double conscience hystérique; elle joue à présent un rôle structurel. Elle tente de rendre compte de l'extrême diversité des symptômes par le morcellement des pensées, des sentiments, des actions. Les associations qui assurent la cohésion de l'ensemble se rompent, et l'incohérence qui en résulte est soumise aux mouvements des affects auxquels ne s'oppose ni le principe de réalité, ni celui d'unité. Le malade se détache du réel et se trouve confronté aux multiples forces intérieures qui colonisent désormais le monde. La prolifération fantasmatique, les hallucinations, toute l'activité délirante se déploie dans un espace déstructuré où les lignes de partage entre soi et le monde sont abolies. Bleuler distingue, au-delà de ce morcellement, une déliaison plus fondamentale au plus intime de l'esprit : «derrière cette Spaltung systématique en complexes idéatifs, nous avons trouvé, antérieurement, un relâchement primaire de la texture associative qui peut conduire à une Zerspaltung incohérente de formations aussi solides que les concepts concrets $»^{5}$. À cette désorganisation, il faut ajouter l'évolution négative de la maladie qui est un critère décisif la rapprochant de la démence. L'ancrage organique, notamment la transmission génétique, reste un débat ouvert. Le succès de la pharmacopée, à partir des années 1950, fait reculer l'analyse bleulerienne. Cette évolution est un retour partiel à Kraepelin, ce que confirme l'approche chosiste qui triomphe avec la classification purement descriptive du Manuel diagnostique et statistique des troubles mentaux ${ }^{6}$.

5 L'aspect technique de la maladie ne relève pas, pour Vestdijk, de l'anecdote. Il s'agit pour lui d'une question de fond ${ }^{7}$. En opposition avec la perspective chrétienne où la 
maladie est une simple confirmation de sa condition, et celle de la Renaissance où l'harmonie et la perfection de l'homme renvoient la maladie à une cause extérieure et, finalement, accidentelle, le romantisme et le positivisme, a priori opposés, développent des affinités profondes dans ce domaine. En absence de visée téléologique, ces courants mènent à une fragmentation de la vision de l'homme qui est devenue constitutive de la modernité au siècle dernier. La portée universelle de la souffrance et de la mort passe désormais par l'exactitude des symptômes et de leur évolution car la culture scientifique, qui est aussi celle du lecteur, devient un vecteur essentiel d'identification. C'est la cohérence du principe de vraisemblance développé au xvire siècle, à présent fondé sur le rationalisme expérimental.

6 Le roman de Vestdijk présente une facture classique, avec un auteur omniscient mais très discret et un récit à la troisième personne. Eddie Wesseling est un jeune garçon venu passer l'été dans une grande ville décrite avec assez de précision pour y reconnaître Amsterdam. Au détour d'une conversation familiale, il apprend que son cousin germain, Fré Bolderhey, souffre d'une maladie mystérieuse: «schizo... schizo... », le terme échappe aux interlocuteurs. Il apprendra le nom de cette maladie le lendemain, lors d'une visite chez tante Bolderhey, et notera immédiatement que dans le mot schizophrénie se trouve la syllabe Fré. Deux choses retiennent son attention: d'une part, son cousin est régulièrement persécuté et battu à l'école ou dans la rue et, d'autre part, s'il est condamné par les médecins, du moins pourrait-il être sauvé à en croire un magnétiseur consulté en désespoir de cause. Ce dernier point intrigue Eddie qui voit l'avenir de Fré compromis et se demande comment l'aider.

7 En fait, la maladie est à l'œuvre dès le début. Du balcon où il prend le frais avec son père, Eddie aperçoit trois hommes en slip de bain qui traversent le quai et plongent dans le canal avant de disparaître rapidement à la nage. Le calme revient. Son père semble n'avoir rien vu, les passants non plus. Un fort sentiment de solitude l'envahit. Dès cet instant, Eddie est pris dans un réseau de thèmes étroitement imbriqués créant une véritable nasse dont il ne peut s'échapper. Le lecteur est lui-même pris au piège car il ne sait jamais quand le réel de référence fait place à l'hallucination. La convention romanesque se retourne contre lui par le jeu d'une écriture dont la précision, servie par une syntaxe rigoureuse, enfante les pires débordements. La figuration de la schizophrénie, mystérieusement précise, présente trois aspects étroitement imbriqués : la dimension notionnelle qui se fait élusive, Eddie cherche des informations sur la maladie dont est frappé son cousin germain mais en obtient rarement; l'idée de "division de l'âme »: esquissée au début du roman, celle-ci donne lieu à une série d'images illustrant la Spaltung et indiquant sa progression; la dernière catégorie, la plus vaste, est celle des visions et altérations diverses et profondes qui s'emparent du garçon.

8 L'enquête du jeune garçon commence par un échec. En jetant son dévolu sur une encyclopédie allemande des années 1910, il n'a aucune chance d'y trouver le terme schizophrénie comme l'indique l'auteur dans une de ses seules interventions directes, "même si la maladie était probablement aussi vieille que l'humanité » (p. 51). L'espoir d'en apprendre plus sur la racine «schizo- » le confronte à une série de termes qui vont nourrir ses hallucinations. Ainsi la ville prussienne de Schkeuditz se métamorphosera en une cité balnéaire envahie par le sable et composée d'innombrables asiles d'aliénés. Plus lourde de conséquences sera la rencontre avec «les Schizopodes, Spaltfüsser en allemand, [qui] constituaient une famille peu nombreuse de petites écrevisses, 
appartenant à l'ordre des Thoracostracées, ressemblant aux crevettes, mais dotés de trois paires de pattes-mâchoires et de cinq paires de pattes thoraciques qui, de plus, étaient fendues» (p. 52). Plus tard, au cours d'un des épisodes les plus hallucinés du roman, Eddie retrouvera une écrevisse agonisant lentement, support d'une compassion sans borne, dans laquelle il reconnaîtra son cousin.

Le sens du mot "schizophrénie" lui sera révélé peu après, durant un épisode de déréalisation qui culminera avec les premières manifestations de dépersonnalisation. La scène se déroule dans une chambre d'enfant dont un mur est tapissé de représentations obscènes, en présence d'une jeune fille - la fille du psychiatre - qui le fascine depuis le début. Son front, qu'elle cachait avec obstination sous un chapeau, s'avère horriblement déformé : les séquelles du rachitisme ou des coups de marteau à réflexes en acier que lui aurait assénés son père. Dans la pièce se trouve aussi un des trois hommes qui s'étaient jetés dans le canal, un étudiant qu'Eddie avait surpris peu avant avec sa mère, ainsi que le psychiatre sous l'aspect d'un énorme singe hurlant des obscénités et menaçant tout un chacun des pires sévices; il est flanqué de deux personnages déjà rencontrés et transformés à présent en d'inquiétants infirmiers. Avec l'assentiment de son maître, un des infirmiers déclare :

- La schizophrénie est la maladie mentale la plus grave qui soit, parce que cette affection ne laisse aucune partie de l'esprit indemne. C'est même sa définition. Schizophrénie signifie littéralement: division de l'esprit; l'esprit se désagrège comme un fagot dont on fait sauter la rouette. Il y a des fagots noirs et des fagots blancs, les blancs sont les pires. Tout le monde souffre, dans une certaine mesure, de schizophrénie parce que l'esprit ne forme jamais une unité au sens strict du mot, il est prédestiné à se désagréger après la mort et doit s'y préparer au cours de la vie ; mais chez ces patients le processus s'accélère et devient plus intense. La partie droite de leur esprit ignore ce que fait la partie gauche; on a parfois l'impression qu'ils s'écartèlent avec autant de violence pour atteindre le plus vite possible le stade de la dissolution générale... (p. 180-181)

10 Les structures psychiques, présentes à travers le récit et les représentations (schéma œdipien réalisé, rapport avec un père distant, sexualité, sentiment d'isolement, déréalisation etc.), jouent le rôle de contraintes destinales contre lesquelles le héros tragique ne peut se battre que s'il les objective, d'où l'importance des images. Par le jeu de l'identification, le lecteur partage le même dérèglement. Pour Eddie comme pour le lecteur, l'hallucination est normale. Le recouvrement partiel de l'expression littéraire et de la schizophrénie est une conséquence du caractère exemplaire de cette maladie car elle porte essentiellement sur le rapport au réel. En tant que théoricien, Vestdijk est attaché à la psychanalyse parce qu'elle assure la primauté du fait psychique, mais le romancier se tourne aussi vers la psychiatrie pour y trouver les descriptions cliniques que l'approche freudienne ne peut offrir. La question de la description n'est pas un simple enjeu littéraire, elle domine l'analyse de la schizophrénie depuis bientôt deux siècles et touche, à travers elle, aux fondements de l'humain.

11 Si le "romancier expérimenté » produit des visions avec plus d'aisance qu'un aliéné, Vestdijk note aussi que ces constructions risquent fort de faire double emploi avec l'œuvre elle-même et qu'en tout état de cause, l'intérêt d'un fou de composition demeure limité ${ }^{8}$. Le parti pris d'exactitude est la conséquence de l'interpénétration des vérités scientifiques et littéraires. La structuration du monde moderne par la science passe aussi par la littérature qui, de son côté, ouvre des possibles où la raison se déploie. C'est toujours un travail par et sur l'imagination. 
12 L'essentiel de la matière romanesque est dominé par un entrelacs de lignes thématiques suivies et proliférantes. Elles reproduisent par l'opposition sous-jacente entre une logique interne (association d'images) et la logique externe (convention sociale) l'isolement du malade. Cet isolement est perçu de l'intérieur, introduisant une relativité qui fait apparaître Eddie comme une figure stable jusqu'à la crise finale. Le noyau originel se révèle dès la deuxième page du récit avec la survenue des trois hommes plongeant dans le canal. À la suite de cette scène, Eddie les imagine se précipitant dans le salon qu'ils époussettent çà et là avec leur slip de bain, puis les voit disparaître dans le bocal à poissons, tenant chacun une figurine en fausse porcelaine.

13 Chaque élément se transformera en un acteur des hallucinations à venir. La statuette représentant une marquise vaguement perverse deviendra sa mère; elle le conduira sur la plage de Schkeuditz où Eddie devra la casser en morceaux pour se sauver lorsqu'elle tentera de le noyer. L'ours en céramique réapparaîtra sous forme de peluche ou de dessins avant qu'Eddie ne devienne lui-même un ours, dansant devant le psychiatre simiesque et ne sentant même plus les coups de cravache. L'élément liquide représente l'attrait et les dangers du sentiment océanique :

Entre ces rochers surtout, les irisations nacrées étaient prodigieuses, et là on voyait également que la mer n'était pas animée par une houle régulière, mais se frayait un passage d'un bassin vers l'autre avec un mouvement changeant et irrégulier, tourbillonnant délicatement: un flux intérieur jouant de toutes les couleurs, les effaçait toutes et toutes les créant de nouveau: du rose, du bleu, de l'orange estompé, un rouge brique exquis, un bleu ardoise, un rouge écrevisse, le vert perlé des lézards, le gris bleu des ailes de pigeons, toutes se fondant les unes dans les autres sans repos ni rupture, comme si la couleur n'était pas couleur, mais gestation de couleur et l'ombre, assurément, l'ombre de la couleur. Cela fluait et tournoyait comme si un cœur aux lents battements insufflait à ces courants un mouvement continuel. (p. 67)

14 C'est un des rares moments où s'apaise l'insupportable tension de l'altérité, celle de Fré habité par l'angoisse et qu'il veut sauver, celle de toutes les créatures qui souffrent parce qu'elles sont au monde, celle du monde enfin qui déchaîne la haine et la violence contre les plus faibles pour mieux se sentir vivre. La fluidité, la confusion des êtres, la continuité entre la matière et la vie sont des sirènes pour qui est sensible à cette douleur infinie, si bien que les être aquatiques semblent habiter les limbes. Placés sur la frontière entre la vie et la matière inerte, ils sont frappés d'une liberté impossible qui les glace : le carrelet possède « l'asymétrie contre nature d'un animal qui, s'étant livré à l'exploration intime de toutes les déformations du corps, ne put retrouver son état premier. " (p. 100) ; l'axolotl est fait pour la vitesse mais reste immobile ${ }^{9}$; l'écrevisse, lointain rejeton de l'encyclopédie allemande, vibre au fond de l'aquarium, le système nerveux rongé par une bactérie. Incarnant la douleur infinie, il représente le cousin malade :

C'était comme si un petit navire de vieil ivoire, ayant fait naufrage, se mourait lentement au fond de la mer, comme si l'on savait que ce navire avait des sentiments et ressentait la douleur, et comme si le partage de cette souffrance résidait bien au-delà de la frontière où la compassion humaine était encore possible, alors que l'on était déjà tourmenté plus vivement qu'au chevet d'une mère malade. Ce n'était pas un être humain qui souffrait, ce n'était même pas un animal, - c'était la matière elle-même qui souffrait plus que ne pourrait souffrir un être animé. Et à quoi lui faisait penser ce tremblement? Cela lui rappelait pourtant quelque chose d'humain - oui, une chose humaine ; si seulement il savait quoi... (p. 172) 
15 Par le jeu des images, la ville entière est menacée de catatonie. Les rumeurs de la circulation estivale évoquent les stations balnéaires, la plage et les dunes ; projetée sur la vision d'une vaste mer pulsante, cette association engendre le spectacle d'une ville envahie par le sable, de rues comblées jusqu'aux toitures, de dunes boulantes interdisant le passage.

16 Le contrepoint à ces motifs prend la forme d'un parapluie noir et menaçant, doué d'une vie propre. Il trouve également son origine au début du roman, dans le récit des persécutions dont Fré, tout jeune encore, est victime à l'école. La mère d'Eddie introduit assez brutalement le sujet : «- C'est quand ils lui ont cassé ce parapluie sur la tête que cela a commencé, non?»(p.10). Eddie voit l'objet pour la première voit lors d'une fantomatique bagarre générale à la sortie d'une école. Le spectre noir survole la mêlée indistincte, cogne mécaniquement les têtes, pique les corps enchevêtrés, se trouve bientôt réduit en lambeaux comme le parapluie de Fré. Cette apparition symbolise la violence originelle. Le parapluie accompagne les tensions les plus vives. Après une longue absence, il réapparaît au moment où Eddie découvre sa mère bras dessus, bras dessous avec l'étudiant et comprend sa capacité à agir sur eux :

Embrasse-la! ordonna-t-il et, non sans maladresse, l'étudiant se pencha vers sa mère, l'embrassa sur les lèvres. Quelques passants s'écartèrent; ils s'embrassèrent une deuxième fois. Prends-la dans tes bras, l'étudiant, vas-y! L'étudiant passa le bras droit autour de la taille de sa mère. Le sang cognait dans les tempes d'Eddie, il s'approcha plus encore, fou d'excitation, il leva le bras droit comme un chef d'orchestre pour leur commander, leur commander, quand tout à coup derrière lui un objet noir se déchaîna, (...) (p. 142)

Le retour du parapluie au moment où Eddie tente d'assouvir un fantasme central par le truchement de l'étudiant - celui-ci lui expliquera d'ailleurs plus tard le mécanisme de cette mise en scène - confirme la confusion croissante entre Eddie et Fré. L'instrument de persécution accablant le schizophrène devient le représentant unique et terrible de toute la violence dont Eddie est l'objet. Le phénomène de dissociation fait que le monde fantasmatique et chaotique du garçon surgit dans le réel et que les forces régulatrices internes se manifestent sous des formes indépendantes et se liguent avec le monde ennemi (gardiens, policiers, psychiatres et infirmier, foule haineuse). Le rôle du parapluie prend d'autant plus de relief qu'il est la seule vision à être reconnue comme telle par Eddie (et le lecteur) sans perdre une seconde sa redoutable efficacité. Le thème du monde ennemi se révèle dans la violence extrême dont les fous sont l'objet. Cette violence est le produit de "la plus intime des unions» entre Fré dans le rôle de la victime (et Eddie qui brûle de prendre sa place) et le monde dans celui du bourreau. Le fou est humilié et torturé et enfermé pour que le monde se sente vivre et jouisse de sa force. L'agressivité atteint son point culminant avec l'irruption du psychiatre anthropoïde qui hurle :

Ils vont numéroter leurs abattis, les dingues, on va les faire passer par les baguettes ; pour les femmes ce sera la ceinture de chasteté en fer, et les hommes, on leur foutra sur la couenne à coups de cravache ; les enfants névropathes et les jolis bébés avec leur petit minois de mongolien, on les noie! Terminé! Plus de complexes dans ma maison! (p. 175)

De délire en hallucination, la violence qui entoure Eddie croît de manière exponentielle. C'est une sorte de lente explosion du psychisme qui transforme les êtres et les choses en ennemis omnipotents. Cette expansion se nourrit d'une vraisemblance restreinte qui se dilate à mesure que le personnage d'Eddie s'effondre. Les fables 
s'enchâssent les unes dans les autres et il n'est rien que le lecteur ne puisse accepter : à la fin du roman, Eddie devient Fré, il crée Cecily, un personnage pourtant très « réel », et s'abîme enfin dans l'indistinct. Lorsque l'éclatement du personnage est parachevé, il n'y a d'autre solution que de changer de perspective. C'est le sens de la rupture que constitue le douzième et dernier chapitre, « La consultation ».

19 C'est un retour brutal à la réalité. Eddie rentre chez lui vers 2 heures du matin; la police a fait des recherches et le rapport semble coïncider avec les aventures du garçon, mais vues de l'extérieur: "On avait vu en divers points de la ville un garçon au comportement étrange, demeurant immobile, appuyé contre un mur, se couchant par terre, faisant de grands gestes ou invectivant les passants, ce qui avait déclenché, à une occasion, une bagarre avec un jeune de son âge.» (p. 228) Le lendemain, son père le conduit chez le psychiatre dont Fré est également le patient. Malgré des propos rassurants - le phénomène n'est pas rare chez les garçons de son âge et presque toujours passager, il n'y a pas de transmission héréditaire -, le médecin doit se rendre à l'évidence. Eddie est en proie, devant lui, à une hallucination. Le garçon détourne le regard vers la fenêtre et voit une épouvantable bagarre sur un trottoir désert. Dans un silence spectral, des jeunes gens se cognent à coups de pieds et de poings, sans cesse ni retenue. Alors surgit, attendue et redoutée, l'incarnation de la terreur. Le parapluie rassemble la violence du monde et se tient prêt à rejeter tout garçon sans défense «dans le royaume innommable dont les portes étaient à jamais scellées par l'absence absolue et méritoire d'humaine compassion. » (p. 237). Cet étrange mérite est repris, dans les dernières lignes du roman, par la réaction du médecin devant l'état du garçon. Il se rend compte que ses efforts seront vains désormais et, d'un geste, invite Eddie à rejoindre son père.

C'était l'attitude d'un homme qui se résignait à l'incompréhension devant des phénomènes dont il percevait la grandeur, sans pouvoir en pénétrer la nature profonde. Qui pourrait lui en vouloir ? Là où les parapluies sont les maîtres du jeu, l'incompréhension est presque une vertu. (p. 238)

Cette fin appelle tout d'abord un commentaire historique car l'impuissance du médecin correspond en partie à la situation de la psychiatrie avant l'apparition de la psychopharmacologie moderne qui commence, en 1952, avec l'emploi du premier neuroleptique, la chlorpromazine ${ }^{10}$. En 1948, Vestdijk a pris position en faveur de l'autonomie du fait psychique à travers l'analyse critique des travaux de Käthe Misch ${ }^{11}$ sur la disparition des symptômes psychiques et somatiques de l'angoisse sous l'effet de l'acétylcholine ${ }^{12}$. Contrairement à l'auteur, Vestdijk considère que l'effet inhibiteur porte non sur l'angoisse mais sur la part subjective de celle-ci, c'est-à-dire sur les effets induits. L'objet de l'angoisse, élément stable, potentiellement accessible à la connaissance, n'a pas disparu. L'intervention chimique masque l'affection sans avoir prise sur elle. L'objet de l'angoisse est constitué par la conscience souffrante à la recherche d'une cause. Cette élaboration, qui n'a souvent aucun rapport avec la cause réelle, est la meilleure voie pour comprendre la nature de ce phénomène omniprésent. Vestdijk part de la notion freudienne de Realangst qui implique un danger extérieur réel, mais l'étend à l'angoisse névrotique dont le caractère indéterminé n'est qu'apparent et transitoire. À tout moment, au cours d'une thérapie par exemple, cette angoisse a vocation à retrouver son objet temporairement refoulé ${ }^{13}$. Le facteur physiologique, accidentel (maladie) ou délibéré (drogue, poison) est subordonné à la dimension psychique. C'est parce que l'angoisse confronte l'individu à une destruction 
totale tout en tendant vers un substrat objectivable, qu'elle pousse l'individu à s'accomplir.

La primauté accordée à l'appareil psychique conduit Vestdijk à s'opposer aux tenants du biologisme (Kurt Goldstein) comme aux philosophes de l'existence (Heidegger, Sartre) qui privent l'homme de sens: "L'individu s'affermit dans l'angoisse pour former un noyau qui se distingue de la manière la plus positive du "Néant" " ${ }^{14}$. Il apparaît comme un défenseur de la psychanalyse par défaut, mais sa seule fidélité est la création. La vérité scientifique, par essence transitoire, vaut comme mode opératoire dans l'élaboration de l'œuvre, sans perdre son statut. Par sa position, Vestdijk maintient la double légitimité scientifique et artistique, et les fond en une unité organique. La prise de position dans le débat autour de la schizophrénie s'exprime par la création littéraire et non par un commentaire intellectuel. Le roman comme représentation et, plus encore, l'image elle-même ont une vertu analytique. Elle tire cette vertu de sa capacité à mobiliser un investissement psychique qui est l'élément fondateur de toute représentation agissante. Elle croche dans l'humain pour aboutir à une condensation de sens où le phénomène de reconnaissance opère.

La rupture que constitue le dernier chapitre par un retour au réel de référence montre à quel point la question de la représentation est problématisée. «Toutes ces choses que tu as vues, que tu devais voir, avaient pour but d'éviter la folie en situant les aberrations en dehors de toi, pour ainsi dire » (p.161) s'entend dire Eddie par une Cecily imaginaire au cours d'un accès hallucinatoire majeur. Le terreau est fertile : les fastes de l'imaginaire déployés avec une rare maîtrise du style et de la composition offrent les ingrédients d'un roman réussi. Reste l'ancrage dans le réel dont Vestdijk fait une condition première et qui rend l'œuvre efficace, pourvue d'une tension propre et agissante sur le lecteur. Si elle ne réside pas dans la mise en scène, où faut-il la chercher?

23 L'écriture est un processus analytique structuré par une tradition formelle. En ouvrant sur l'espace social, celle-ci contraint à l'objectivation. Le dispositif, évoqué plus haut, des trois positions - le médecin, le patient, l'écrivain - réalise un mode de fonctionnement caractéristique du roman moderne depuis le xix ${ }^{\mathrm{e}}$ siècle. La science détermine les conditions de recevabilité et imprime des orientations. La douleur s'impose comme une extension du sujet vers l'inconnu, elle crée la tension nécessaire à toute coagulation du sens. L'écrivain occupe une position excentrée. Il doit saisir dans une forme publique une énergie qui lui échappe. La dialectique unissant les trois forces détermine le degré de liberté de l'œuvre par rapport à la norme. Le théoricien pragmatique qu'est Vestdijk demeure plus sensible à la nécessité d'une forme donnée qu'à l'inertie des conventions. Il exploite donc le caractère attendu du roman pour en inverser l'effet et porter son interrogation sur les fondements du fait littéraire. Le thème de la schizophrénie constitue un cas particulier mais significatif de ce travail.

La littérature moderne est centrée sur la part inconnue de l'homme et exerce à travers ce déséquilibre une emprise dont les poètes maudits ont été une manifestation célèbre. Ce lien d'emprise est l'aspect intériorisé des rapports de force analysés par Pierre Bourdieu $^{15}$, aboutissant à l'émergence d'un nouvel espace littéraire. La continuité entre la structuration psychique et la structuration sociale se fait à travers la notion de praxis qui caractérise toute création artistique. L'œuvre soude ce que les analyses disjoignent, répondant à des filiations différentes. C'est la liberté mise en œuvre. 

expériences nouvelles. L'exploration des lointains intérieurs fait de l'anormal une vertu, si bien que l'art et la science se découvrent des causes communes. La littérature et la médecine se réunissent pour essayer les psychotropes, avec la folie pour tiersarbitre. Le psychiatre Moreau (1804-1884) symbolise ce rapprochement et les tensions qu'elle fait naître. Son ouvrage le plus célèbre, Du haschisch et de l'aliénation mentale ${ }^{16}$, témoigne d'une compréhension nouvelle de la nature des perceptions et de leur cohérence, mais aussi d'une orientation organiciste induite par l'approche pharmacologique. Il a commencé une étude approfondie des effets du haschisch lors d'un voyage en Orient et, convaincu de la similitude entre le rêve, la folie et les effets de la drogue, développe une thérapeutique basée sur l'emploi du haschisch. Ses recherches passent par un usage personnel régulier et par l'observation de volontaires, médecins ou écrivains. Par l'entremise de Théophile Gautier, nombre de poètes et de romanciers se réunissent régulièrement; ce sera le Club des Hachichins (1844-1849) dont les activités sont supervisées par Moreau. Son apport essentiel est l'idée qu'il existe à l'origine des troubles mentaux une «modification intellectuelle primitive, toujours identique à elle-même » :

C'est une désagrégation, une véritable dissolution du composé intellectuel que l'on nomme facultés morales. (...) Le résultat est le même dans l'ordre spirituel et dans l'ordre matériel : la séparation, l'isolement des idées et des molécules dont l'union formait un tout harmonieux et complet ${ }^{17}$.

(anifestations si variées de la folie ne sont que les «signes extérieurs » de cette atteinte centrale. On reconnaît les trait pertinents retenus par Bleuler pour décrire la schizophrénie, mais l'identité entre les ordres matériel et spirituel est plus qu'une comparaison. Pour Moreau la «désassociation» des idées a toujours un fondement purement organique ${ }^{18}$.

Baudelaire s'est par la suite moqué, sans le nommer, du docteur Moreau et de sa thérapeutique : «Le fou qui prend du hachisch contracte une folie qui chasse l'autre, et quand l'ivresse est passée, la vraie folie, qui est l'état normal du fou, reprend son empire, comme chez nous la raison et la santé. (...) Le médecin qui a inventé ce beau système n'est pas le moins du monde philosophe $»^{19}$. Claude Pichois attribue la pique au "peu de goût qu'avait Baudelaire pour le hachisch $»^{20}$, mais cet ennemi du Progrès a dû sentir la menace que représente la réduction du rêve à un phénomène physique : le nivellement de la poésie par les hommes en redingote. L'influence des thèses de Moreau sur la psychiatrie s'étendra jusqu'au $\mathrm{xx}^{\mathrm{e}}$ siècle et forcera les poètes à jouer le rôle d'un véritable contre-pouvoir.

Inversement, la pensée scientifique exerce sa capacité critique au sein même des lettres comme le montre l'exemple d'André Breton. La découverte de la psychiatrie, lors de son internat, a joué un rôle important au moment où il abandonne une vision de la poésie marquée par Mallarmé et Valéry pour concevoir un mouvement expérimental et anti-littéraire ${ }^{21}$. L'apport du surréalisme à la compréhension des phénomènes mentaux n'est pas une irruption de la littérature dans un domaine étranger, mais la rencontre de finalités critiques. dont l'émergence remonte au xvi ${ }^{\mathrm{e}}$ siècle. Le schème de base apparaît vers 1550 avec l'abandon d'une conception collective de la littérature. L'idée antique de l'inspiration divine, réappropriée, ne distingue désormais plus les poètes de la masse des ignorants, 
mais le poète singulier et conscient de lui-même des versificateurs en tous genres. Par ce coup de force, le poète sort d'un univers bâti sur un nombre fini de textes se ramifiant en d'infinies interprétations pour se confronter à ce qui lui échappe radicalement ; l'œuvre devient ainsi une projection dans l'avenir. Le classicisme, qui n'aimait ni l'incertitude ni l'individu, voulut faire de sa doctrine une science sûre conduisant immanquablement à la réussite l'auteur respectueux des règles, mais la rupture était consommée. Sous un néoplatonisme de convention, une filiation voit le jour qui se prolonge jusqu'à nos jours, faisant de la poésie une expérience de la transcendance. De Ronsard écrivant que «les vers viennent de Dieu, / et non de l'humaine puissance ${ }^{22}$ " à nos grands contemporains, les mots et les concepts changent mais pas l'état de réceptivité qui résulte d'un long travail sur soi. Ascèse laïque qui, de la "vertu précieuse» de jadis au travail analytique contemporain, demeure une plongée «Au fond de l'Inconnu pour trouver du nouveau ». L'élan consacre la liberté et la dépendance du poète, de tout artiste. Cette sujétion à ce qui n'a forme ni parole traverse toute la poésie depuis un siècle et demi. Peletier écrivait déjà, à l'époque où le mot inspiration prenait son sens actuel : «Car les Muses ne se veulent point avoir par force : il faut attendre le Dieu à venir : il faut épier cette sainte chaleur. Combien de fois se trouvera le Poète tout interdit, tout dépourvu, tout mal à son commandement : bref, tout autre que soi-même? $»^{23}$.

Dans une pareille perspective, la continuité entre la folie et la littérature s'explique par la proximité de l'abîme et un sens commun de la destruction. Contrairement à ce que l'on a pu penser, ce n'est pas l'œuvre qui est ouverte, mais la littérature elle-même. L'inspiration - l'attente inquiète, le surgissement - est devenue le lien ombilical avec le grand Autre dont la schizophrénie fouille aujourd'hui l'attache. Face à la Gorgone reine, l'écrivain avance et se protège par la maîtrise du verbe. La formation de la représentation qui sous-tend ce roman puise sa force dans l'homologie de structure dont le modèle est fourni par la compassion infinie qui habite le personnage. Le sentiment est d'autant plus vif et absolu que, ne trouvant à s'assouvir, il engendre une violence dont l'extension la nourrit en retour.

31 La confrontation à la douleur ou la détresse d'autrui donne lieu à un mouvement naturel de sympathie qui peut aller jusqu'à cette «fatigue de compassion » qui désigne le traumatisme secondaire produit par des situations extrêmes et répétées. Dans le roman, ce même mouvement apparaît comme la seule explication de la maladie puisque les causes classiques, l'hérédité, le contexte familial ou social sont écartées. Elle devient le fil conducteur de la raisonnante déraison et connaît un double couronnement : après un long processus, Eddie se transforme d'abord en son cousin Fré, parachevant son désir sacrificiel, puis il se désagrège tout à fait. Cette crise, donnée pour un point de non-retour de la maladie, marque la frontière entre les domaines $d u$ romanesque et ce sur quoi les mots n'ont plus prise. Le roman joue le rôle de médiateur entre le désastre de la schizophrénie et la confrontation avec le grand Autre.

La forme majeure de cette expérience est la mort spirituelle et physique. Dans la définition de la schizophrénie donnée dans le roman, chacun souffre, à des degrés divers, de la maladie puisque : «l'esprit ne forme jamais une unité au sens strict du mot, il est prédestiné à se désagréger après la mort et doit s'y préparer au cours de la vie » (p. 180). Ce qui est abordé du point de vue de la psyché recouvre la question de la finitude souvent formulée en termes philosophiques et ancrée au cœur de la littérature $\mathrm{du} \mathrm{xx}^{\mathrm{e}}$ siècle. Un exemple significatif de rupture avec l'idéalisme littéraire, en 
l'occurrence celui du surréalisme d'après guerre, est le premier grand recueil d'Yves Bonnefoy, Du mouvement et de l'immobilité de Douve (1953). L'exergue est tiré d'un passage de la Phénoménologie de l'esprit de Hegel: «Ce n'est pas cette vie qui recule d'horreur devant la mort et se préserve pure de la destruction, mais la vie qui porte la mort, et se maintient dans la mort même, qui est la vie de l'esprit. L'esprit conquiert sa vérité seulement à condition de se retrouver soi-même dans l'absolu déchirement. » La philosophie et la psychologie se rencontrent dans la réflexion sur le fondement de la création en tant que pratique. L'interrogation n'appartient en propre ni à l'une, ni à l'autre. Dans la littérature française, marquée par une forte théorisation, on emprunte la voie conceptuelle pour construire le rapport au réel, alors que, dans la tradition plus libre et moins médiatisée de la littérature néerlandaise, c'est l'expérience du lien donné qui prime. L'image développe alors une force opératoire qui lui confère une pleine légitimité. C'est sans doute pour cette raison que l'on aime souligner, aux Pays-Bas, le caractère hollandais de l'œuvre de Simon Vestdijk, et qu'ailleurs on se méprend sur le sens du prosaïsme que le qualificatif suggère. De son parcours de la pensée du $\mathrm{xx}^{\mathrm{e}}$ siècle, Vestdijk retient qu'il n'est de salut que dans l'expérience particulière. Il oppose au Néant la schizophrénie qui est, de la manière la plus concrète, la mort à l'œuvre et maintenue au cœur de la vie. La leçon du parapluie mêle l'art et la vie : si l'on ne peut vaincre l'angoisse, du moins est-il possible de la localiser et de la circonscrire dans une forme, ce qui est aussi une poétique. Si l'incompréhension peut avoir une vertu, comme le suggère la fin du roman, c'est qu'elle favorise le cycle qui relie l'angoisse à l'expérience de la beauté pour retourner à l'angoisse de nouveau. Le mysterium tremendum est l'idéal tragique et vital de l'art.

« 3 trois hommes nus, D. Margadant » est l'intuition d'une fracture dont le roman est l'exploration. De redding van Fré Bolderhey relate le naufrage d'un jeune homme, mais l'énergie libérée domine l'œuvre et affermit le postulat résolument pratique que l'auteur formule ainsi : "c'est un postulat de l'angoisse elle-même, qui s'attacherait encore à un objet si tous les objets du monde eussent été détruits ${ }^{24}$. " Angoisse et pulsion créatrice sont parfois interchangeables.

\section{NOTES}

1. Hans Visser, Simon Vestdijk, een schrijversleven [S.V., une vie d'écrivain], Utrecht, Kwadraat, 1987, p. 328, 341 et 416.

2. Simon Vestdijk, De redding van Fré Bolderhey, Amsterdam, De Bezige Bij, 1948 ${ }^{1}, 1973^{5}$.

Toutes les traductions sont de l'auteur de ces lignes ; la traduction du roman sera publiée par les éditions Phébus en 2004.

3. Simon Vestdijk, Het wezen van de angst [L'essence de l'angoisse], Amsterdam, De Bezige Bij, 1979, p. 661.

4. L'édition des œuvres, parues entre 1974 et 1990, comprend les romans (52 vol.), l'œuvre poétique ( 3 vol.), les nouvelles ( 1 vol.) et les essays sur la musique (10 vol.). Il faut ajouter à cet ensemble un volume posthume de poèmes (1986) et 26 essais ou recueils d'articles. 
5. Cité in : J. Laplanche et J.-B. Pontalis, Vocabulaire de la psychanalyse, Paris, P.U.F., 1967, 199412, p. 435.

6. Diagnostic and Statistical Manual of Mental Disorders, classification des maladies mentales publiée par l'Association américaine de psychiatrie (la quatrième édition date de 1994).

7. Simon Vestdijk, De zieke mens in de romanliteratuur [L'homme malade dans la littérature romanesque], Amsterdam, De Bezige Bij, 1964 ${ }^{1}$, $1977^{2}$.

8. Idem, p. 94.

9. Le nom de cet amphibien se reproduisant à l'état larvaire est généralement lié à Xolotl qui désigne en nahuatl le dieu aztèque des déformations et de la mort.

10. Sous le nom de Largactil, ce produit reste un des antipsychotiques les plus utilisés.

11. Het wezen van de angst, p. 352-356.

12. Le physiologiste allemand Otto Loewi a mis en évidence son rôle en 1921, prouvant la transmission chimique de l'influx nerveux. C'est le premier neurotransmetteur connu.

13. Het wezen van de angst, p. 313-339. Laplanche et Pontalis, Op. cit., donnent pour traduction «Angoisse devant un danger réel ».

14. Idem, p. 677.

15. Pierre Bourdieu, Les règles de l'art. Genèse et structure du champ littéraire, Paris, Seuil, 1992.

16. Jacques-Joseph Moreau (de Tours), Du haschisch et de l'aliénation mentale, études psychologiques, Paris, Librairie de Fortin, Masson et Cie, 1845.

17. Idem, p. 36.

18. Il ne s'agit pas de lésions décelables, mais d'une « altération de la sensibi-

lité, c'est-à-dire l'action irrégulière, exaltée, diminuée, pervertie, de ces propriétés spéciales d'où dépend l'accomplissement des fonctions intellectuelles. » Idem, p. 397.

19. Charles Baudelaire, « Du vin et du hachisch » (1851), CEuvres complètes, édition Claude Pichois, 2 vol., Paris, Gallimard, Bibliothèque de la Pléiade, 1975, vol.1, note p. 397.

20. Ibid., p. 1371.

21. André Breton (1896-1966) commence des études de médecine en 1913. En 1915, il devient interne dans le service de neuropsychiatrie de l'armée à Saint-Dizier, puis à la Pitié dans le service de Joseph Babinski (1857-1932), élève de Charcot et créateur de la neurochirurgie, et enfin au Val-de-Grâce et à Saint-Mammès.

22. Pierre de Ronsard, "Ode à Michel de l'Hopital ", cité par Grahame Castor in : La poétique de la Pléiade. Étude sur la pensée et la terminologie du $\mathrm{XVI}^{e}$ siècle, trad. Yvonne Bellenger, Paris, Honoré Champion, 1998 (1964ㄴ), p. 56.

23. Jacques Peletier, Art poétique, 1555, in : Sébillet, Aneau, Peletier, Fouquelin, Ronsard, Traités de poétique et de rhétorique de la Renaissance, introduction, notices et notes de Francis Goyet, Le Livre de Poche, 1990, p. 228.

24. Het wezen van de angst, p. 679. Italiques de l'auteur.

\section{RÉSUMÉS}

Dans son roman De redding van Fré Bolderhey [Le salut de F. B.], Simon Vestdijk (1898-1971) relate l'évolution de la schizophrénie chez un jeune garçon de 16 ans. La compassion extrême que celuici ressent envers un cousin germain plus âgé, lui-même schizophrène avéré et persécuté à ce titre par ses camarades, joue un rôle essentiel dans la progression inéluctable de la maladie. 
La question de la forme est centrale dans la compréhension des rapports entre cette maladie insaisissable, définie par Bleuler en 1911 et symbolisant la folie au xxe siècle, et la littérature conçue comme un processus exploratoire.

Après avoir étudié comment le romancier structure l'œuvre autour des hallucinations du personnage principal, l'article aborde les relations intimes que la littérature noue avec la médecine en établissant le lien avec la notion d'inspiration apparue au xvie siècle. La confrontation avec la science conduit à une prise de position en faveur du fait psychique et à une méditation sur l'importance non de la cause de l'angoisse, mais de son objet. Le roman se nourrit de la tragédie que représente la schizophrénie pour exemplifier la transformation de l'angoisse en énergie créatrice.

Simon Vestdijks Roman De redding van Fré Bolderhey [Die Rettung des Fré Bolderhey] beschreibt die unaufhaltbare Entwicklung der Schizophrenie bei einem sechzehnjährigen Jungen. Sein extremes Mitleid mit einem etwas älteren Vetter, der selbst schizophren und deswegen der Bosheit seiner Kameraden ausgeliefert ist, spielt eine wesentliche Rolle im Fortschreiten seiner eigenen Krankheit.

Die Frage der Form ist von grundlegender Bedeutung für das Verständnis der Beziehungen zwischen dieser unfassbaren, von Bleuler 1911 beschriebenen Krankheit, die den Wahnsinn im 20. Jahrhundert symbolisiert, und Literatur, als Untersuchungsprozess verstanden.

Im Aufsatz wird zuerst analysiert, wie der Romancier das Werk um die Halluzinationen der Hauptfigur herum anlegt. Dann wird die enge Verknüpfung zwischen Literatur und Medizin untersucht, wobei dieVerbindungen mit dem im 16. Jahrhundert auftretenden Begriff der Inspiration in den Vordergrund treten. Die Konfrontation mit der Naturwissenschaft führt zu einer Stellungnahme zugunsten der psychischen Fakten und nicht zu einer Reflexion über die Gründe der Angst, sondern über deren Gegenstand. Der Roman lebt von der Tragödie, welche die Schizophrenie darstellt, um die Umwandlung der Angst in schöpferische Energie zu illustrieren.

\section{AUTEUR}

\section{SPIROS MACRIS}

Université Charles-de-Gaulle - Lille 3 